\title{
Changes to the law on consent in South Africa: implications for school-based adolescent sexual and reproductive health research
}

\author{
Melanie Zuch ${ }^{1 *}$, Amanda J Mason-Jones ${ }^{2}$, Catherine Mathews ${ }^{3}$ and Lesley Henley ${ }^{4}$
}

\begin{abstract}
Background: The National Health Act, No 61, 2003 in South Africa is the first effort made by the government to protect health-related research participants under law. Implemented on March 1, 2012, the law mandates active consent from a parent or legal guardian for all research conducted with research participants under the age of 18 years. This paper focuses on the Act's implications for school-based adolescent sexual and reproductive health research.

Discussion: Although well intentioned, the added legal protections in the National Health Act may have the unintended consequence of reducing participation rates in school-based adolescent sexual and reproductive health research, thereby excluding the most at-risk students. The Act may also compromise adolescents' right to dignity and privacy, especially considering the personal nature of research on sex and sexuality. Devolved, discretionary decision-making, which empowers local human research ethics committees to permit a wider range of protective measures, including passive consent, independent adolescent consent or community consultation ought to be considered. The continued and direct involvement of young people in their sexual and reproductive health and well-being is an important principle to uphold.

Summary: This paper calls for a re-examination of section 71's ethical guidelines relating to informed consent in the National Health Act, No 61, 2003 in South Africa in order to better serve the interests of South African adolescents in sexual and reproductive health research.
\end{abstract}

\section{Background}

Protecting the welfare of research participants, particularly vulnerable groups such as adolescents, is of primary importance to any researcher to ensure that research-related harm and exploitation are avoided. However, a recent audit commissioned by the Vulnerable Subjects Working Group of the National Health Research Ethics Council (NHREC) found that prior to the implementation of the National Health Act No 61, there was limited specific legal protection for vulnerable research participants in South Africa [1]. Before the implementation of the Act, the legal framework did not specifically address health-related research with adolescents or other vulnerable groups at all, leading research

\footnotetext{
* Correspondence: melanie.zuch@gmail.com

'Brown University, Medical Research Council, PO Box 19070, Cape Town, Tygerberg 7505, South Africa

Full list of author information is available at the end of the article
}

ethics committees to rely on guidance from general principles such as laws relating to the medical treatment of 『minors'.

It would seem then that the National Health Act No 61 of 2003 [2] and its research-specific provisions (s71), enacted on March 1, 2012, would be welcomed wholeheartedly by researchers, but a closer look at the provisions contained therein belies the potentially farreaching consequences for conducting school-based adolescent health research in South Africa. The purpose of this paper is to explore these proposed new provisions in terms of the effects on adolescent health research and to suggest that these provisions do not take into account the emerging autonomy of the adolescent sufficiently nor the current South African social context and make suggestions that will optimize the balance between adolescents' access to research and their protection as research subjects.

\section{Biomed Central}




\section{The South African regulatory framework}

Prior to March 1, 2012, the framework governing research in South Africa permitted adolescents to consent independently to take part in research. In -Ethics in Health Research: Principles, Structures and Processes," the South African Department of Health states that adolescents, defined as persons who have reached puberty, may consent unassisted to research so long as: (i) the research poses -no more than minimal risk" to the adolescent; (ii) -the parents or legal guardians or community at large are unlikely to object" to the adolescent's participation; (iii) the protocol justifies -why adolescents should be included as participants"; and (iv) the protocol justifies -why the adolescent participants should consent unassisted" [3]. In contrast, the provision of Section 71 of the National Health Act No 61, 2003, does not make the same distinctions between children and adolescents with regards to independent consent. The Act is the first effort made by the South African government to protect research participants under law [4]. Section 71 of the Act mandates active written consent from a parent or legal guardian for all research conducted with subjects under the age of 18 . The Act says: -where research or experimentation is to be conducted on a minor for a therapeutic purpose, the research or experimentation may only be conducted-(a) if it is in the best interests of the minor; (b) in such manner and on such conditions as may be prescribed; (c) with the consent of the parent or guardian of the child; and (d) if the minor is capable of understanding, with the consent of the minor." For non-therapeutic research and experimentation, it may only be conducted, -(i) in such manner and on such conditions as may be prescribed; (ii) with the consent of the Minister (of Health); (iii) with the consent of the parent or guardian of the minor; and (iv) if the minor is capable of understanding, the consent of the minor" [2]. No other caregiver or custodian will be able to give consent for a child's participation in research [5].

Thus, active consent from a parent or legal guardian is the only way that subjects under the age of 18 can participate in research in South Africa [2].

Of particular concern is wide definition of -health research" in the Health Act. According to the definition, -health research" includes (among others) -any research which contributes to knowledge of the biological, clinical, psychological or social processes in human beings" [2]. Arguably, social science research will then need to meet similar consent requirements as those laid down for medical research.

While the intentions of the National Health Act are to provide legal protection to research participants, the Act leaves no room for independent adolescent consent for studies exploring their sexual and reproductive health.
Indeed, the mandate of active parental consent under the Act may greatly reduce participation rates and introduce recruitment bias to school-based adolescent sexual and reproductive health research projects.

\section{Consent for adolescent sexual and reproductive health research}

The issue of participant informed consent remains central to any research project in the medical and social sciences. The fundamental principle stated in the Declaration of Helsinki is a person's right to make an autonomous informed decision about his or her participation in research [6] whilst the first point in the Nuremberg Code deems informed consent to be -absolutely essential" [7]. However, ambiguity surrounds questions of who can consent for young adolescents, what type of consent is most appropriate, and at what point that young person can consent for him or herself [8]. In the process of protecting adolescents from exploitative or undesired research, there exists a tension between the protection of research subjects, and excessive regulations that may disrespect the autonomy of the individual, or result in such research being unfeasible [9].

This debate is especially acute in adolescent sexual and reproductive health research [10], which is often conducted to develop and evaluate effective interventions to reduce sexual risk behavior. Indeed, the scientific and ethical review group at the World Health Organization states in their guidelines for adolescent reproductive health research that omitting this type of research can -perpetuate inadequate understanding of the particular reproductive health needs of adolescents and result in failure to deliver adequate services to this group" [11]. This type of research typically takes the form of surveys or classroom-based interventions and is conducted with high school students who are in general 12 years and older. Such studies have been deemed to be no more than minimal risk; in other words, the risks associated with daily life or routine medical and psychological examinations. Indeed, recent research shows that most adolescents do not report increased distress when completing surveys which address sensitive topics, which include questions about suicidal behavior, illicit drug use and physical and sexual abuse [12].

\section{Issues in parental consent}

There are generally two primary forms of parental consent used in social science and health services research with adolescents. Active consent necessitates a Đyes' response from the parent, in order that a young person may participate in research. Passive consent assumes the parent's affirmative response, unless the parent indicates otherwise $[13,14]$. It is assumed that active consent comes with greater confidence that a parent has actually 
read and understood the details of the research project, whereas with passive consent, a parent's lack of response could result from their failure to receive, read or understand the letter rather than their willingness to allow the child to participate [13]. Active consent is also assumed to bring a sense of parental involvement in the research project, which can be important to interventions that aim to reduce rates of adolescent sexual risk behavior and/or to change social norms. However, active parental consent-often denoted by a signature on a form-is not always a true indication of an adult's understanding of or involvement in the research in question $[15,16]$.

A study in South Africa demonstrated that despite an apparent active parental consent response rate of $94 \%$, only $65 \%$ of parents had received the information letter and consent form during a follow-up interview. Many parents also reported being unaware of the letter's contents, despite having signed the consent form. Follow up interviews were conducted with 18 parents who denied consent for their child to participate; 15 of these parents actually did want their children to participate. Of the 12 parents who were interviewed after not responding to the letter sent home, all 12 had not received the consent letter, and said they also wanted their child to participate in the research[16]. It is not surprising therefore, that active parental consent can generate low response rates of only $30 \%-60 \%$ [14]. But it may not necessarily reflect parents actively refusing to allow their children to participate in the research. Oftentimes non-response is a result of a parent's not receiving the letter, or a lack of understanding [17].

Failure to understand an informed consent letter may be a particular problem in South Africa, where high illiteracy rates are prevalent $[18,19]$. Parents may not feel comfortable signing or finger printing a document that they cannot fully comprehend or their signature may not be a valid sign of their comprehension [16]. Beyond literacy rates, cultural and language differences can impede informed consent, as can the effect of a power imbalance between the researcher and the subject, and subject's parent [19].

Requiring active parental consent procedures may also hinder research by adding a prohibitive cost to research $[20,21]$ and by introducing a potentially significant sample bias into the data $[17,22]$. A study of parental consent procedures in the United States demonstrated that students who gained consent from their parents were more likely to be female, White, from intact homes, less likely to smoke cigarettes, and have parents who were more likely to be educated [14]. In this way, students at greater risk for poor health are likely to be disproportionately excluded by active consent procedures $[13,14]$. This sample bias not only poses a problem for research integrity; it also may prevent research from reaching the students who stand most to benefit from it.
The new Act specifically mandates consent from a legal guardian, despite the fact that many children in South Africa do not live with a parent or a legal guardian, but rather with another caretaker or custodian. According to a report by the South African Human Rights Commission and UNICEF, only $32 \%$ of South African children live with both of their biological parents and 19\% have lost one or both parents [23]. Additionally, UNICEF estimates that there are 3.7 million orphans in South Africa [24], and according to a study by the South African Institute of Race Relations, 98,000 children were living in child-headed households as of 2008 [25]. Whilst the exact number of children without a legal guardian is unknown, these statistics suggest that the number is high, as children who have lost their parents may be living under the auspices of another adult who has not legally adopted them.

We suggest that laws governing research in South Africa must consider its unique social circumstances. The National Health Act will prevent children without a legal guardian from accessing research that could be of great benefit to them in terms of reducing rates of sexual risk behavior, pregnancy and STI/HIV transmission. This is especially problematic given that orphans and vulnerable children, precisely those without legal guardians, are at an elevated risk of contracting HIV and other STDs, and becoming pregnant [26].

\section{Independent adolescent consent}

Early empirical evidence has shown that young adolescents may be as competent as adults in their ability to provide informed consent in terms of -stringent legal standards of competency" [27]. While an adolescent's ability to make competent decisions does vary from person to person, this evidence compels a deeper look into the option of independent consent for school-based research with adolescents aged from 12 years, particularly if procedures are in place for research staff to gauge the subjects' levels of understanding. Such an assessment may include verbal or written tests to gauge the participant's level of understanding of the consent form, understanding of confidentiality procedures, and knowledge of their rights as research subjects as well as their rights to withdraw [28].

In some cases, children as young as 8 years old have been deemed competent to make autonomous decisions in medico-legal matters [29] and from 5 years in research [30]. An adolescent's ability to provide informed consent can depend on individual emotional maturity level, their perceived or actual ability to make decisions in their day-to-day lives (which can be dependent on cultural and/or familial norms), reasoning skills, memory and language [29]. Interestingly, the Children's Act of South Africa sets the minimum age of independent consent for medical treatment and surgical operations at 12 years [31]. If consistent standards were 
applied to adolescent participation in research, active parental consent would not be mandatory as long as the subject were deemed able to consent for him or herself. Passive parental consent or community-based collaborative consent may mitigate any potentially negative consequences of active procedures, and potentially grant adolescents more autonomy during research participation. This is particularly important in research into adolescent sexual and reproductive health, which oftentimes includes sensitive and personal topics that an adolescent may not want to share with his or her parents.

Adolescents are in the process of becoming fully autonomous individuals [32] and although an adolescent's degree of autonomy and cognitive ability to consent will differ from individual to individual, it is generally accepted that 12-year-olds are old enough to make autonomous and informed decisions about their participation in a school-based adolescent sexual research project which poses minimal risk to them [27].

\section{Discussion}

Requiring active parental consent may not be appropriate for all study settings and populations, particularly where cultural norms differ from Western ethical standards [19]. Concerns with the new guidelines are not limited to the tangible ways in which they may hinder the implementation of research. In prohibiting students aged 1218 years from providing independent consent under any circumstances, the enactment of Section 71 of the National Health Act No 61, 2003 may be compromising a child's right to dignity and privacy $[4,33]$. Confidentiality is a central tenant of research ethics, especially in research surrounding sensitive topics such as sexuality. In South Africa in particular, discussions surrounding sexuality are often shrouded in stigma [34] and parentchild communication with regards to sex and sexuality is often limited [32]. An adolescent therefore may not feel comfortable confronting a parent or guardian about participation in a sexual and reproductive health research study or may face disapproval if he or she chooses to do so [32]. If a parent interprets an adolescent's desire to participate in a sexual and reproductive health study as proof that their child is sexually active, the child could lose access or knowledge of sexual and reproductive health services due to stricter parental supervision [10].

We believe that adolescents from the age 12 onwards should be allowed to participate in school-based sexual and reproductive health research, which recognizes and seeks to understand their unique set of needs in order to develop programs to keep them healthy even if their parents would choose to deny them access on moralistic grounds. It is important to note that according to current South African research ethics guidelines any human research must receive prior approval by a relevant human research ethics committee in order to ensure an adequate balance of risks and benefits regardless of whether their parents consented to their participation. Moreover, in the case of school-based research, the approval of the Department of Education and the school principal is also needed before implementation.

Social realities in South Africa must be reflected in laws relating to research among adolescents about their sexual and reproductive health. The Department of Health's prior ethical guidelines [3] appear sufficient whilst the new changes appear to be inconsistent with other guidance and are retrogressive. It is acknowledged that parental involvement is critical to an adolescent's wellbeing, and programs to improve adolescent sexual and reproductive health should actively involve parents in the planning stages and encourage improved parent-child communication.

Several researchers have rightly asserted that age is a very limited measure of an adolescent's cognitive ability to fully understand and consent to research $[29,30]$ and that adolescents are developmentally more inclined to underestimate the effects of taking a risk, and thus cannot necessarily be deemed capable of making informed decisions about participation in research [34]. In this regard, other methods of obtaining collaborative adult consent must be considered, including obtaining active parental consent at the beginning of the school year during registration to better ensure comprehension and response [16], as well as methods that embody a process of community engagement whose principles are found at the heart of rural communities in South Africa [35,36]. One possibility is to consult community forums to gain consent for research projects, instead of gaining consent from parents only [35,37]. Such group consent procedures can facilitate the process of decision-making, ensuring that the study is understood and supported by the community as well as by individual participants [38]. Significantly, this principle of community consultation already underpinned the Department of Health's prior ethical guidelines. Other alternatives include the inclusion of adolescents on research ethics committees [39], and/or the establishment of Youth Advisory Committees, in which adolescents themselves can inform the development, content and implementation of research $[10,40]$.

\section{Summary}

Adolescent sexual and reproductive health research in South Africa is of great importance, as teenage pregnancy, sexually transmitted disease and HIV-rates remain high in this population [41]. Maintaining high standards in our ethical approach is critical to protect vulnerable research subjects from potential harm. However, to preserve the right of young people to self-determination, we call for a re-examination of the National Health Act guidelines for research. The proposed 
enactment may actually undermine the rights of young people in South Africa.

\author{
Author details \\ 'Brown University, Medical Research Council, PO Box 19070, Cape Town, \\ Tygerberg 7505 , South Africa. ${ }^{2}$ Adolescent Health Research Unit, University of \\ Cape Town, Specialist Scientist, Health Systems Research Unit, Medical \\ Research Council, PO Box 19070, Cape Town, Tygerberg 7505, South Africa. \\ ${ }^{3}$ School of Public Health and Family Medicine, University of Cape Town, \\ Health Systems Research Unit, Medical Research Council, PO Box 19070, \\ Cape Town, Tygerberg 7505, South Africa. ${ }^{4}$ School of Child and Adolescent \\ Health, University of Cape Town, Cape Town, Rondebosch 7700, South \\ Africa.
}

\section{Authors' contributions}

AMJ conceived of the idea, and MBZ researched and drafted the first version of the paper. All authors revised for content and style, and all have read and approved the final manuscript.

\section{Competing interests}

$\mathrm{MZ}$ is a law student, AMJ and CM are involved in adolescent health research, AMJ was previously a member of a research ethics committee and $\mathrm{LH}$ is a current member of a research ethics committee.

Received: 3 May 2011 Accepted: 10 April 2012 Published: 10 April 2012

\section{References}

1. National Health Research Ethics Council (NHREC) Ethical-legal protection for vulnerable research participants in South Africa. An Audit of relevant laws and ethical guidelines. 2011 [http://www.nhrec.org.za/wp-content/ uploads/2011/ethicalguidelines.pdf].

2. National Health Act, 2003. Government Gazette Republic of South Africa; 2000.

3. Ethics in Health Research: Principles, Structures and Processes Pretoria: Department of Health; 2004.

4. Strode A, Grant C, Slack C, Mushariwa M: How well does South Africa's National Health Act regulate research involving children? SAMJ 2005, 95:265-268.

5. Slack C, Strode A, Fleisher T, Gray G, Ranchod C: Enrolling adolescents in HIV vaccine trials: reflections on complexities from South Africa. BMC Medical Ethics 2007, 8:1-8.

6. Declaration of Helsinki: Ethical Principles for Medical Research Involving Human Subjects World Medical Association; 2008

7. Trials of War Criminals before the Nuremberg Military Tribunals under Control Council Law Washington, D.C.: U.S. Government Printing Office; 1949.

8. Gallagher M, Haywood SL, Jones MW, Milne S: Negotiating informed consent in school-based research: a critical review. Children and Society 2010, 24:471-482.

9. Levine R: Research involving adolescents as subjects. Ann N Y Acad Sci 2008, 1135:280-286.

10. Flicker S, Guta A: Ethical approaches to adolescent participation in sexual and reproductive health research. $J$ Adolesc Health 2008, 42:3-10.

11. World Health Organization: Reproductive health involving adolescents. [http://www.who.int/reproductivehealth/topics/ethics/ adolescents_guide_serg/en/index.html].

12. Langhinrichsen-Rohling, Arata C, O'Brien N, Bowers D, Klibert J: Sensitive research with adolescents: just how upsetting are self-report surveys anyway? Violence and Victims 2006, 21:425-444.

13. Ross JG, Sundberg EC, Flint KH: Informed Consent in School Health Research: Why, How, and Making it Easy. J Sch Health 1999, 69:171-176.

14. Tiggs BB: Parental consent and adolescent risk behavior research. $J$ Nursing Scholarship 2003, 35:283-289.

15. Waggoner WC, Sherman BB: Who understands? II: A survey of 27 words, phrases, or symbols used in proposed clinical research consent forms. IRB: Review of Human Subjects Research 1996, 18:8-10.

16. Mathews C, Guttmacher SJ, Flisher AJ, Mtshizana Y, Hani A, Zwarenstein M: Written Parental Consent in School-Based HIV/AIDS Prevention Research. Am J Public Health 2005, 95:1266-1269.
17. Courser MW, Shamblen SR, Lavrakas PJ, Collins D, Ditterline P: The impact of active consent procedures on nonresponse and response error in youth survey data. Eval Rev 2009, 33:370-395.

18. UNDP: Human Development Report 2009: South Africa [http://hdrstats.undp. org/en/countries/country_fact_sheets/cty_fs_ZAF.html].

19. Marshall PA: Informed consent in international health research. J Empir Res Hum Res Ethics 2006, 1:25-42.

20. Esbensen FA, Miller MH, Taylor T, He N, Freng A: Differential Attrition Rates and Active Parental Consent. Eval Rev 1999, 23:316-335.

21. Secor-Turner M, Sieving R, Widome R, Plowman S, Vanden Berk E: Active parent consent in health surveys with urban middle class school students: processes and outcomes. I SchHealth 2010, 80:73-79.

22. Baker JR, Yardley JK, McCaul K: Characteristics of responding-, nonresponding- and refusing-parents in an adolescent lifestyle choice study. Eval Rev 2001, 25:605-618.

23. South African Human Rights Commission/UNICEF: South Africa's Children: a Review of Equity and Child Rights. 2011.

24. UNICEF: Protection for Orphans and Vulnerable Children.[http://www. unicef.org/southafrica/protection_6633.html].

25. Holborn L, Eddy G: South African Institute of Race Relations: First Steps to Healing the South African Family. 2011.

26. The United States President's Emergency Plan for AIDS Relief: Orphans and Vulnerable Children.[http://www.pepfar.gov/strategy/ prevention_care_treatment/133367.htm].

27. Weithorn LA: Children's Capacities to Decide About Participation in Research. IRB: Ethics and Human Research 1983, 5:1-5.

28. Bruzzese JM, Fisher CB: Assessing and Enhancing the Research Consent Capacity of Children and Youth. Applied Developmental Science 2003, 7:13-26.

29. Fundudis T: Consent issues in medico-legal procedures: how competent are children to make their own decisions? Child and Adolescent Mental Health 2003, 8:18-22.

30. Danby S, Farrell A: Opening the research conversation. In Ethical Research with Children. Edited by: Farrell A. Maidenhead: Open University Press; 2005:49-67.

31. Children's Act, 2005. Government Gazette Republic of South Africa; 2006.

32. Lesch E, Kruger L-M: Mothers, daughters and sexual agency in one lowincome South African community. Soc Sci Med 2005, 61:1072-1082.

33. Risjord M, Greenberg J: When IRBs Disagree: Waiving Parental Consent for Sexual and reproductive health Research on Adolescents. IRB: Ethics and Human Research 2002, 24:8-14.

34. Campbell C, Foulis CA, Maimane S, Sibiya Z: "I have an evil child at my house": stigma and HIV/AIDS management in a South African community. Am J Public Health 2005, 95:808-815.

35. Mkabela Q: The Afrocentric Method in Researching Indigenous African Culture. The Qualitative Report 2005, 10:178-189.

36. Kamwangamalu NM: Ubuntu in South Africa: A sociolinguistic perspective to a Pan-African concept. Critical Arts 1999, 13:24-41.

37. Jaspan HB, Doka NF, Mathews C, Flisher AJ, Mark D, Middelkoop K, Wood R, Bekker L-G: A qualitative assessment of perspectives on the inclusion of adolescents in HIV vaccine trials in South Africa. Int J STD AIDS 2010, 3:172-176.

38. Schrag B: Research with Groups: Group Rights, Group Consent, and Collaborative Research. Sci Eng Ethics 2006, 12:511-521.

39. Coyne I: Research with Children and Young People: The Issue of Parental (Proxy) Consent. Children and Society 2010, 24:227-237.

40. Royal College of Paediatrics and Child Health ': This House believes that we have gone too far in granting young people the responsibility for making decisions about their own health care': record of a debate held in the Ethics and Law session of the RCPCH Annual Meeting, York 2009. Arch Dis Child 2011, 96:123-126.

41. National Antenatal Sentinel HIV and Syphilis Prevalence Survey in South Africa, 2009 Pretoria: Department of Health; 2010.

\section{Pre-publication history}

The pre-publication history for this paper can be accessed here: http://www.biomedcentral.com/1472-698X/12/3/prepub

doi:10.1186/1472-698X-12-3

Cite this article as: Zuch et al: Changes to the law on consent in South Africa: implications for school-based adolescent sexual and reproductive health research. BMC International Health and Human Rights 2012 12:3 